\title{
DEFORMATION AND DAMAGE MECHANISMS DURING THERMOMECHANICAL FATIGUE OF A SINGLE-CRYSTAL SUPERALLOY IN THE $<001>$ AND $<011>$ DIRECTIONS
}

\author{
Mikael Segersäll ${ }^{1}$, Johan J. Moverare ${ }^{1,2}$, Kjell Simonsson ${ }^{1}$, Sten Johansson ${ }^{1}$ \\ ${ }^{1}$ Linköping University, Dept. of Management and Engineering, SE-58183 Linköping, Sweden \\ ${ }^{2}$ Siemens Industrial Turbomachinery AB, Materials Technology, SE-61283 Finspång, Sweden
}

Keywords: single-crystal superalloy, thermomechanical fatigue, deformation mechanisms, twinning, shearing

\begin{abstract}
The purpose of this paper is to investigate the differences in mechanical response and microstructural behavior when the single-crystal Ni-based superalloy CMSX-4 is subjected to thermomechanical fatigue (TMF) in two different crystallographic directions, $<001>$ and $<011>$. An out-of-phase (OP) straincontrolled TMF cycle with $R_{\epsilon}=-\infty$ in the temperature range 100 to $850^{\circ} \mathrm{C}$ was used. As expected, the material exhibited, when loaded in the $<001>$ direction, a higher number of cycles to failure compared to the $<011>$ direction, when equivalent strain ranges were compared. High strain ranges led to crystallographic fractures along one of the $\{111\}$ planes while low strain ranges led to non-crystallographic fractures. This result was valid for both $<001>$ and $<011>$ directions. Specimens with random fractures also showed recrystallization close to the fracture surface. Twinning was found to be a major deformation mechanism for most specimens. A change in deformation mechanism from twinning to shearing was found in specimens subjected to loading in the $<011>$ direction when going from low to high strain ranges. This investigation also indicated that crack propagation is a consequence of recrystallization and not the other way around.
\end{abstract}

\section{Introduction}

Nickel-based superalloys are used as blade material in gas turbines and aero engines due to their excellent resistance to mechanical and environmental degradation at elevated temperatures [1, 2], see Figure 1 for a singlecrystal turbine blade. Components used in these hightemperature applications are often subjected to both cyclic loads and cyclic temperatures up to $1100^{\circ} \mathrm{C}$. Due to this, isothermal fatigue tests are not necessarily sufficient when trying to predict life times for components in high temperature applications. Therefore, TMF-studies are of interest for turbine and aero industry. A better understanding of deformation and damage mechanisms that occur during TMF is required to be able to make reliable lifetime predictions for turbine blades and thereby prevent blade failure. By using single-crystal superalloys instead of poly-crystal superalloys creep properties at elevated temperatures are improved. Ni-based superalloys have the characteristic $\gamma / \gamma^{\prime}$-microstructure where $\gamma$ acts as matrix and the cuboidal $\gamma^{\prime}$-precipitates act as strengtheners. The $\gamma^{\prime}$-phase is rich of $\mathrm{Al}$ and $\mathrm{Ti}$ with an $L 1_{2}$ ordered structure.

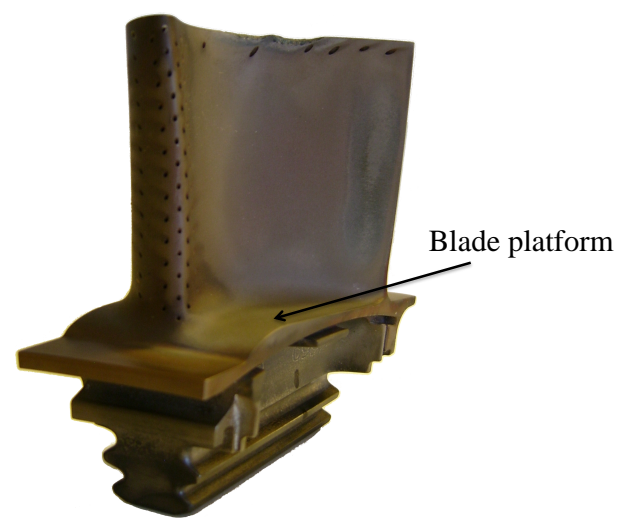

Figure 1: A single-crystal turbine blade. Courtesy of Siemens Industrial Turbomachinery.

The single-crystal superalloy CMSX-4 is a second generation superalloy widely used as blade material in gas turbines. Single-crystal turbine blades are commonly made from investment casting where the single-crystal structure is obtained by directional solidification. When casting single-crystal turbine blades it is often only the primary direction, i.e. $<001>$, that is controlled while the secondary direction is randomly oriented. Most common is to have the $<001>$ crystallographic direction in the upward direction of the single-crystal turbine blade, since this direction has the lowest stiffness $\left(\mathrm{E}_{<001>}=125 \mathrm{GPa}\right.$ compared to $\mathrm{E}_{<011>}=220 \mathrm{GPa}$ [3]) and therefore possesses the best fatigue properties, but also due to the fact that the $<001>$ is the easiest direction to solidify since it has the highest growth rate when solidifying single-crystals. 
This paper is a continuation of the work performed by Moverare et al. [4] where TMF deformation and damage mechanisms up to $1000^{\circ} \mathrm{C}$ were investigated for virgin and aged CMSX-4 material respectively. In that study the material was loaded in the $<001>$ crystallographic direction only. In this paper on the other hand, a comparison between two loading directions will be made. The $\langle 011\rangle$ crystallographic direction is of interest since cracks on the blade platform (see Figure 1) are often visible. The propagation of these cracks is affected by the properties in different crystallographic directions where the $<011>$ direction is one of the most critical directions.

Research concerning TMF behavior in Ni-based single-crystal superalloys is not that common in literature, and the existing research mostly deals with materials loaded in the $<001>$ crystallographic direction $[5,6,7]$. Comparisons of TMF behavior for different crystallographic directions are rarely seen, even though comparisons concerning other properties are sometimes found. For example tensile testing at temperatures from $20-900{ }^{\circ} \mathrm{C}$ in the $<001>,<011>$ and $<111>$ crystallographic directions was done for the SRR99 superalloy [8]. The results showed an orientation dependent yield strength, i.e a non Schmid behaviour. Also differences in tensile properties at room temperature between $<100>,<110>$ and $<111>$ directions have been studied [9]. One conclusion drawn was that yielding of the Ni-based single-crystal superalloys was controlled by propagation of deformation bands. The deformation bands are in turn dependent on dislocation cross-slip and how the $\gamma$-channels are oriented compared to the loading direction. Regarding the creep behavior in the $<011>$-direction, it was in [10] reported that localized macroscopic deformation bands were visible on specimens subjected to creep at $750^{\circ} \mathrm{C}$ under $800 \mathrm{MPa}$, but at higher temperatures the deformation was less localized. Another investigation concerning creep showed that the $<011>$-direction has a high creep rate [11]. This was explained by few activated slip systems and stress concentrations in the $\gamma$-matrix. Dislocation structures from cyclic testing in room temperature in two crystallographic directions have been investigated in [12]. In the [011] crystallographic direction the dislocation structures were grouped in structure bands while in the [111] crystallographic direction the dislocation structures looked like walls extending themselves perpendicular to the tensile axis.

The two major aims with this investigation are (i) to compare the results with the previous study by Moverare et al. [4] where the same material was
TMF tested using a higher maximum temperature and (ii) to investigate the differences in mechanical response and microstructural behavior between the two crystallographic directions $<001>$ and $<011>$.

\section{Experimental Procedure}

For this study the Ni-based single-crystal superalloy CMSX-4 with chemical composition Ni-5.65Al-9.6Co6.4Cr-0.11Hf-0.61Mo-2.9Re-6.6Ta-1.02Ti-6.4W (wt.\%) was used. Out-of-phase (OP) TMF testing was performed in the temperature range $100-850^{\circ} \mathrm{C}$ using a MTS servo-hydraulic TMF machine with induction heating and forced air cooling. OP TMF testing means that temperature and load are cycled out-of-phase, i.e. with tensile stresses at low temperatures and compressive stresses at high temperatures. Figure 2 shows a simplified version of the temperature-strain cycle used.

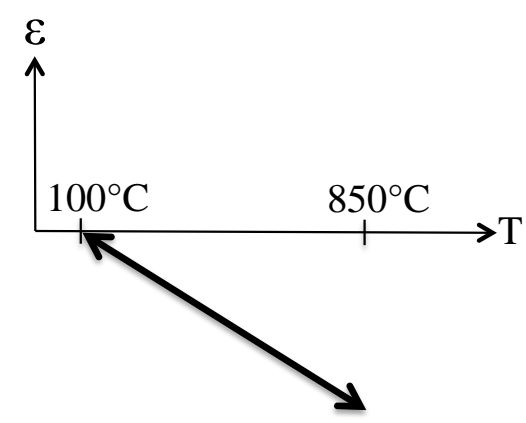

Figure 2: Temperature-strain cycle during the OP TMF tests.

In total, eight specimens with circular cross sections and diameters of $6.35 \mathrm{~mm}$ were tested, four in each different crystallographic direction, <001> and $<011>$. All tests were performed with a strain ratio of $\mathrm{R}=\epsilon_{\min } / \epsilon_{\max }=-\infty$ with a hold time of $5 \mathrm{~min}$ applied at maximum temperature. The strain ranges used were chosen in order to obtain realistic life times compared to a real component, for example, a gas turbine blade. As such, $<001>$ specimens were subjected to generally higher strain ranges compared to the $<011>$ specimens, since they have a lower stiffness. After the TMF tests, the specimens were examined by stereomicroscope before they were cut parallel to the loading direction for further microstructural investigation by scanning electron microscopy (SEM). The SEM samples were prepared by grinding and mechanical polishing, but no samples were etched. The following microstructure investigation was performed in a Hitachi SU70 SEM, using 
acceleration voltages from 10 to $20 \mathrm{kV}$. Finally, by use of an electron backscatter diffraction (EBSD) system by HKL technology orientation imaging microscopy (OIM) was performed.

\section{Experimental Results}

\section{TMF Behaviour}

Results from the OP TMF testing are shown in Figures 3-4. In Figure 3 mechanical strain ranges are plotted against cycles to failure $\mathrm{N}_{f}$, where the black squares represent specimens loaded in the <001> direction and white squares represent specimens loaded in the $\langle 011\rangle$ direction. As expected, high mechanical strain ranges result in a low number of cycles to failure while low mechanical strain ranges results in a high number of cycles to failure. It can be seen that the material when tested in the $<001>$ direction shows a higher number of cycles to failure compared to tests in $<011>$ direction when equivalent mechanical strain ranges are compared. This is expected since CMSX-4, like other single-crystal superalloys [13], shows lower stiffness in the $\langle 001\rangle$ direction compared to the $<011>$ direction. The specimens in this experiment showed the stiffnesses of $\mathrm{E}_{<001>}=124-130 \mathrm{GPa}$ and $\mathrm{E}_{<011>}=222-229 \mathrm{GPa}$, respectively. A material with lower stiffness generally shows better fatigue properties in strain-controlled testing and therefore encounter a higher number of cycles to failure.

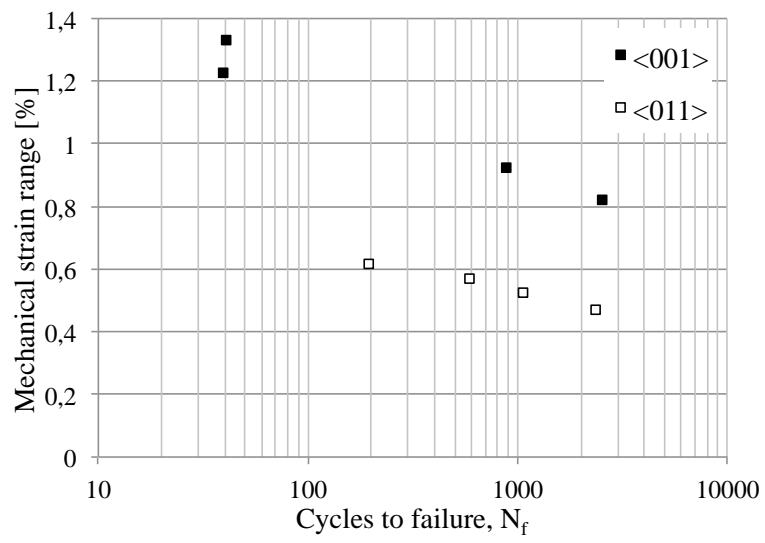

Figure 3: Mechanical strain range versus cycles to failure $\mathrm{N}_{f}$.

In Figure 4 peak stresses at half life are plotted versus cycles to failure. Notable is that stress levels are similar for both crystallographic directions and that the maximum stress level shows a smaller influence on

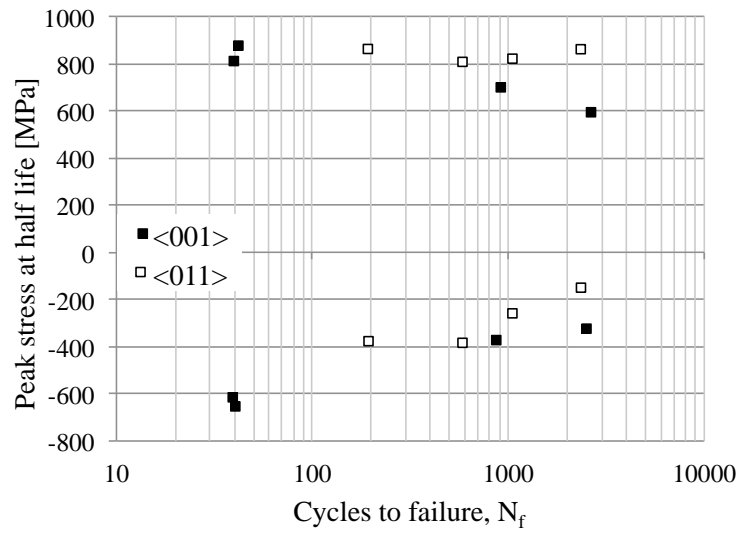

Figure 4: Peak stress at half life versus cycles to failure $\mathrm{N}_{f}$.

$\mathrm{N}_{f}$ for the $<011>$ direction compared to the $<001>$ direction. An interesting result for the specimens loaded in the $<011\rangle$ direction is that the peak stress at half life decreases up to 600 cycles to failure but then increases. This behavior was not expected, but a difference in deformation mechanism may explain the behavior and this is further discussed in the Discussion section. A different behavior between tests with high and low strain ranges for the $<011>$ direction can also be seen in Figures 5-6, where the evolution of maximum and minimum peak stress are plotted as a function of cycles. For the test with high strain range, $\Delta \epsilon=0.61 \%$, the stresses are fairly constant during the test, while for the test with a low strain range, $\Delta \epsilon=0.47 \%$, both the maximum and minimum stress shifts more to the tensile side during the test.

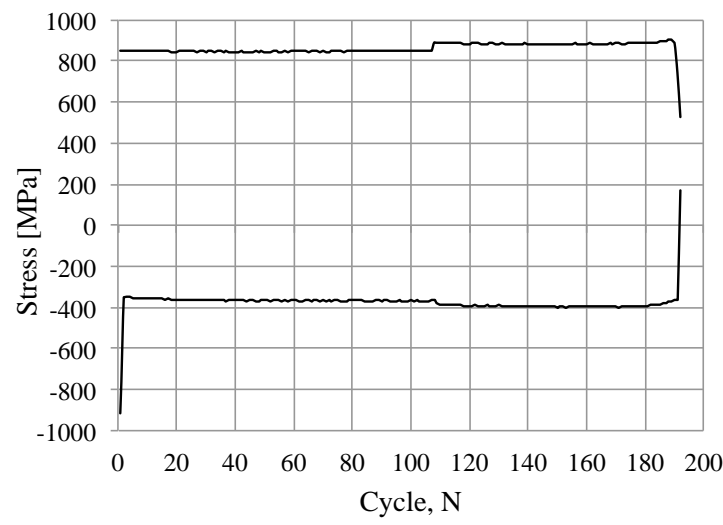

Figure 5: The evolution of stress with cycles for a $<011>$ specimen subjected to high strain range, $\Delta \epsilon=0.61 \%$. 


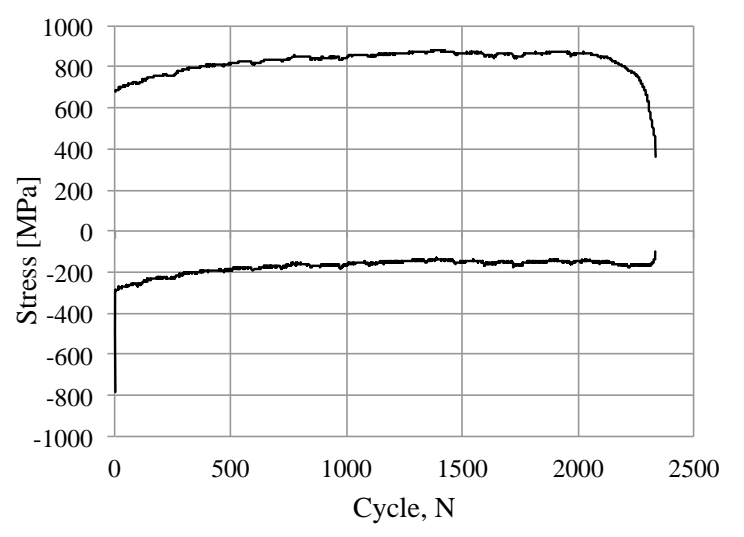

Figure 6: The evolution of stress with cycles for a $<011>$ specimen subjected to low strain range, $\Delta \epsilon=0.47 \%$.

\section{Microscopy}

Virgin CMSX-4 microstructure consists of cuboidal $\gamma^{\prime}$ precipitates surrounded by a $\gamma$-matrix. No extended rafting (directional coarsening of the $\gamma^{\prime}$-particles) was visible after the TMF testing and this was not expected since rafting is mostly activated at temperatures above $900^{\circ} \mathrm{C}[14]$. When examining the specimen fracture surfaces it was notable that high strain ranges led to crystallographic fractures while low strain ranges instead led to non-crystallographic fractures. This result was valid for both crystallographic directions, <001> and $<011>$.

$<001>$ Direction Specimens loaded in the $<001>$ direction showed at high strain ranges fractures along one of the $\{111\}$ crystallographic planes, Figure $7(\mathrm{a})$. This is an indication that these planes are favored for both crack initiation and crack propagation. For specimens subjected to lower strain ranges, instead non-crystallographic fractures were observed, hence the fractures have occurred more randomly, Figure 7(b).

A typical microstructure for $<001>$ loaded material, subjected to high strain ranges, showed extensive twinning parallel to the crystallographic fracture, i.e. parallel to one of the $\{111\}$ crystallographic planes, see Figure 8 . The twins were widely spread throughout the specimen. That twinning really had occurred was confirmed by the EBSD technique where the misorientation profile showed a misorientation of $60^{\circ}$ between the twins and the matrix. Furthermore, in other areas precipitation of topologically close-packed (TCP) phases was also observed within the twins.
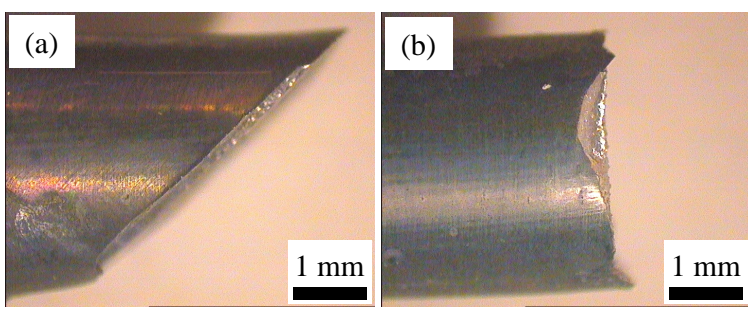

Figure 7: Fractured specimens that were loaded in $<001>$ direction, (a) high strain range, $\Delta \epsilon=1.33 \%$ and (b) low strain range, $\Delta \epsilon=0.82 \%$.

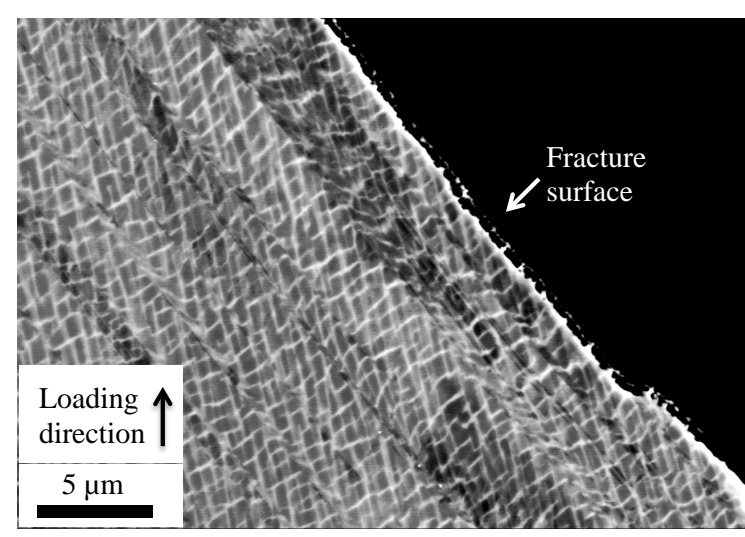

Figure 8: A backscattered electron image of $<001>$ loaded material subjected to a high strain range, $\Delta \epsilon=1.33 \%$. Twins parallel to the crystallographic fracture are visible.

Low strain ranges instead led to non-crystallographic fractures of the specimens, see Figure 9 for an illustration of the microstructure close to the fracture surface. In this case recrystallization close to the fracture surface was observed. Even if the fracture was non-crystallographic, extensive crystallographic deformation was found. Twinning and formation of TCP phases along crystallographic planes out from the fracture surface are also visible in Figure 9. The TCP phases occur as bright spots in the backscattered electron image. Also recrystallization of the microstructure was observed within the twins.

In addition to the crack where fracture eventually occurred other secondary cracks were found on specimens subjected to both high and low strain ranges. A severe plastic deformation of the microstructure around these cracks was found. Furthermore, recrystallization, twinning and precipitation of TCP phases were also visible, see Figure 10. Twinning was once again confirmed by the use of the EBSD technique; the 


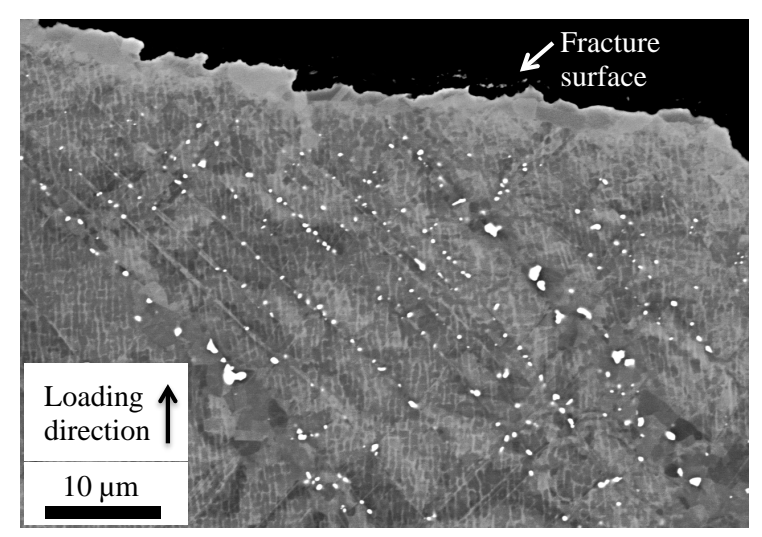

Figure 9: A backscattered electron image of $<001>$ loaded material subjected to a low strain range, $\Delta \epsilon=0.82 \%$. Crystallographic deformation close the noncrystallographic fracture, TCP phases appear as bright spots.

misorientation profile in Figure 11 shows a difference of $60^{\circ}$ between the twin and bulk material. Similarly, as close to the fracture surface, deformation such as twinning was found to be crystallographic along one of the $\{111\}$ planes. In Figure 11 recrystallization is also confirmed and the recrystallized grains appear as fields with different colors.

$\leq 011>$ Direction The fracture surface appearance for specimens loaded in the $<011>$ direction was similar to that for specimens loaded in the $<001>$ direction, i.e. high strain ranges led to crystallographic fracture along one of the $\{111\}$ planes, while low strain ranges resulted in non-crystallographic fractures, see Figure 12(a)-(b). Specimens subjected to medium strain ranges showed a mixed fracture mode where the fractures occurred mainly along one of the $\{111\}$ crystallographic planes but also non-crystallographic parts of the fractures were visible. In this case fractures have occurred at different $\{111\}$ planes leading to a serrated fracture.

Material subjected to low strain ranges in the $<011>$ direction showed significant recrystallization at the fracture surface, see Figure 13. No clear crystallographic fracture was found; instead the fracture had occurred more randomly. Also here twinning was found to be a major deformation mechanism, see Figure 14. Inside the twins TCP phases have precipitated.

Microstructure investigation of the specimen loaded in the $<011>$ crystallographic direction at high strain range shows a more localized deformation at much fewer deformation bands compared to any other speci-

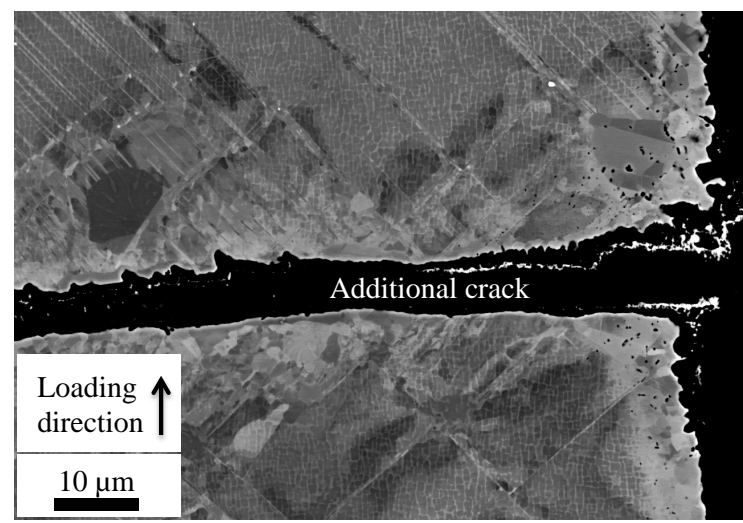

Figure 10: A backscattered electron image of $<001>$ loaded material subjected to a low strain range, $\Delta \epsilon=0.82 \%$. Crystallographic deformation surrounding an additional crack which was located below the fracture surface.

men. Twinning was no more the dominant deformation mechanism, instead shearing of the microstructure was dominant.

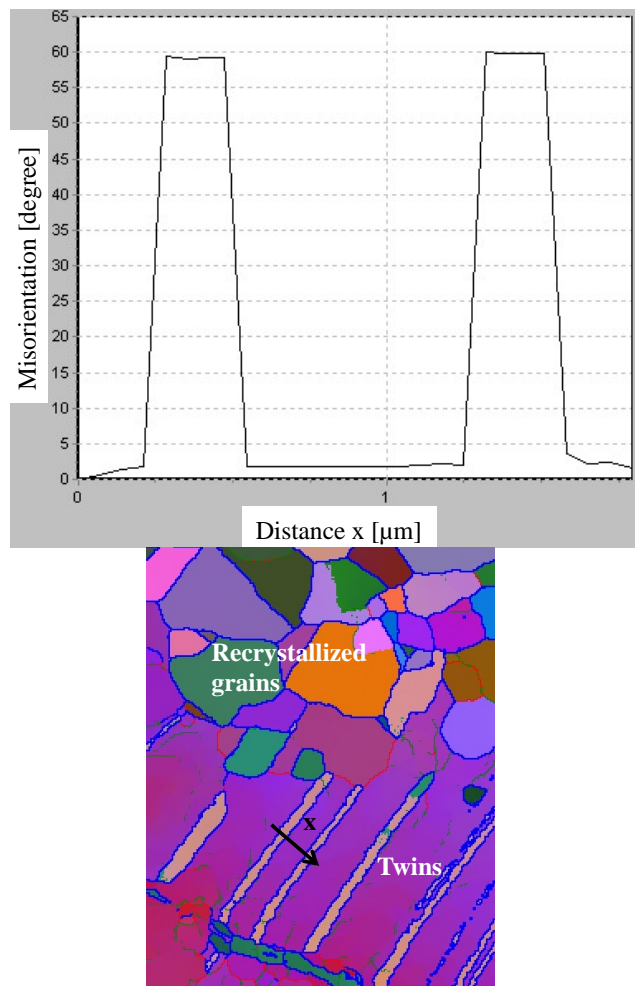

Figure 11: EBSD map and orientation profile for a specimen subjected to load in the $<001>$ direction at low strain range, $\Delta \epsilon=0.82 \%$. 

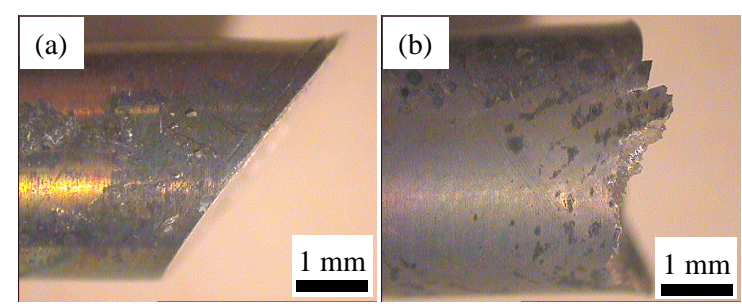

Figure 12: Fractured specimens that were loaded in $<011>$ direction, (a) high strain range, $\Delta \epsilon=0.61 \%$ and (b) low strain range, $\Delta \epsilon=0.47 \%$.

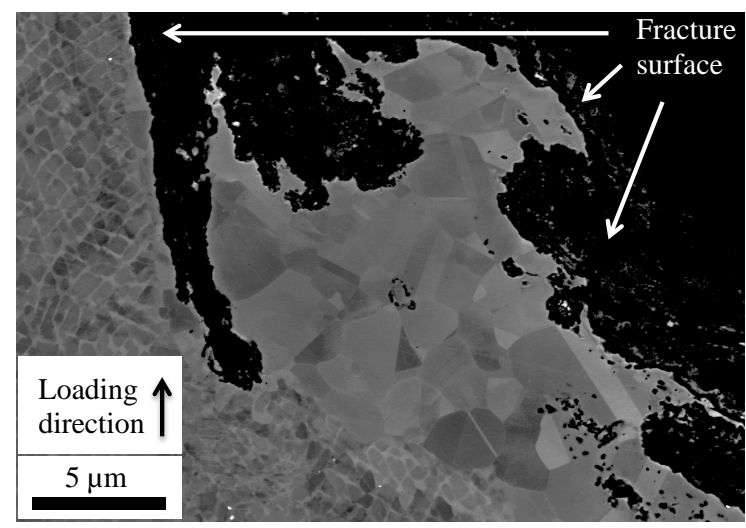

Figure 13: A backscattered electron image of $<011>$ loaded material subjected to a low strain range, $\Delta \epsilon=0.47 \%$. Recrystallization close to the fracture surface.

The sequence of images shown in Figure 15 show one deformation band stretching through the specimen from one side to the other. Figure 15(a) shows how the $\gamma / \gamma^{\prime}$-microstructure is sheared close to the side of the specimen. EBSD mapping showed that there were small orientation differences (around $10^{\circ}$ ) between the sheared deformation band and the matrix. This proves that this deformation band is not a twinning phenomena. Further into the specimen the deformation band becomes more and more sheared, see Figure 15(b). Also here EBSD mapping showed a small crystallographic misorientation between the sheared deformation band and the bulk material. The sheared deformation band later becomes recrystallized and precipitation of TCP phases is visible within the recrystallized deformation band, Figure 15(c). The last picture shows the other side of the specimen where a crack has initiated at the recrystallized deformation band, Figure 15(d). Figure 16 shows another type of deformation mechanism for a specimen subjected to TMF in the $<011>$ direction at a medium strain range. Here it is visible that

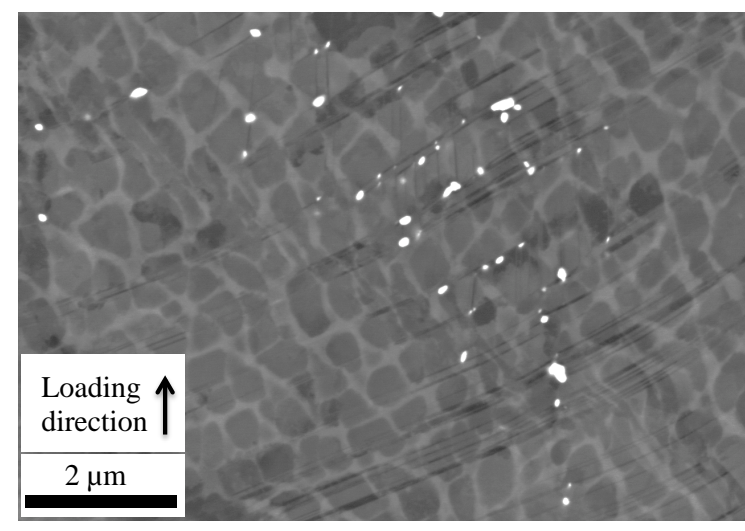

Figure 14: A backscattered electron image of $<011>$ loaded material subjected to a low strain range, $\Delta \epsilon=0.47 \%$. Twinning and precipitation of TCP phases.

the $\gamma / \gamma^{\prime}$-microstructure has been sheared by a number of slip bands cutting the $\gamma^{\prime}$-particles. The SEM investigation for this specimen also showed deformation structures, such as twinning, at different $\{111\}$ planes compared to the final fracture.

\section{Discussion}

Research concerning TMF of single-crystal superalloys is not common in the literature. Even more rare is to find the differences in TMF behavior between different crystallographic directions and the deformation and damage mechanism that occur. This gives the background and motivation to the present paper.

The CMSX-4 material subjected to high strain ranges showed crystallographic fractures along one of the $\{111\}$ planes while material subjected to low strain ranges showed non-crystallographic fractures; this result was valid for both $<001>$ and $<011>$ directions. Crystallographic fractures along the $\{111\}$ plane have been reported previously both for tensile testing at high temperature [8] and cyclic loading at room temperature [15]. Even if specimens subjected to low strain ranges showed non-crystallographic fractures, crystallographic deformation such as twinning were visible within the microstructure. Previous research concerning OP TMF in the $<001>$ direction for CMSX-4 material has shown that twins on the $\{111\}$ planes were formed ahead of the crack tip and therefore formed a preferable path for crack propagation [16]. That conclusion seems to be valid also in this work.

Precipitation of TCP phases during TMF in CMSX-4 has been reported before [4]. This work confirms that 

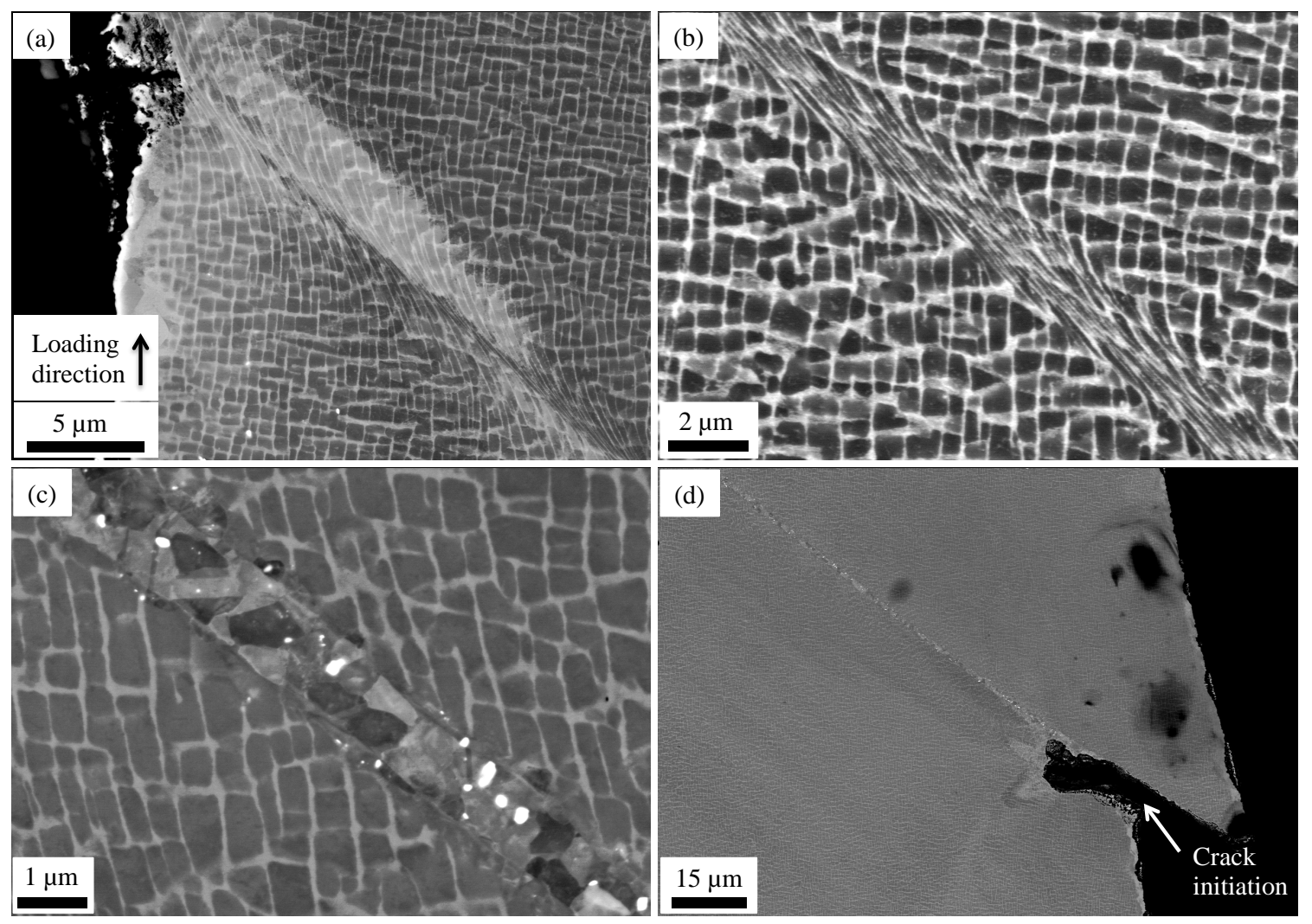

Figure 15: Backscattered electron images of $<011>$ loaded material subjected to high strain range, $\Delta \epsilon=0.61 \%$. Pictures of one deformation band stretching through the specimen. (a) Shearing of the $\gamma / \gamma^{\prime}$-microstructure, (b) increased shearing, (c) recrystallization and (d) a crack has initiated at the recrystallized deformation band.

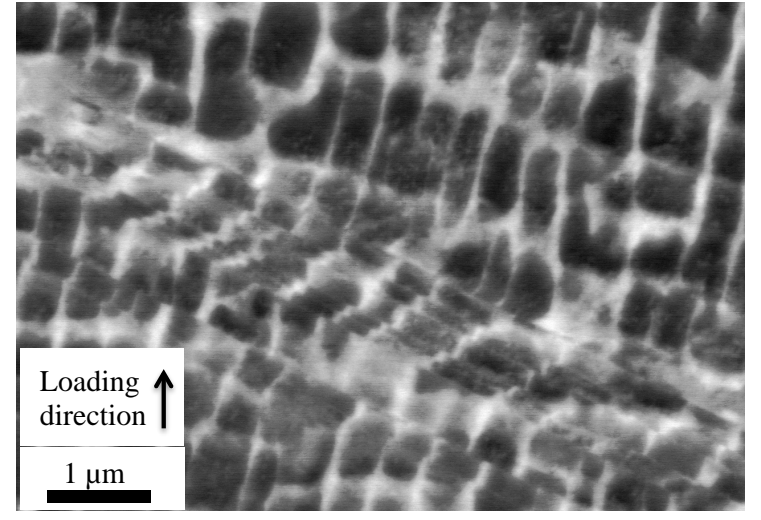

Figure 16: A backscattered electron image of $<011>$ loaded material subjected to a medium strain range, $\Delta \epsilon=0.52 \%$. Slip of the $\gamma / \gamma^{\prime}$-microstructure.

the bright spots in Figures 9 and 14 are TCP phases. One can also conclude that they are a $\mu$-type TCP phase.

Recrystallization close to the fracture surface was found for specimens subjected to low strain ranges, see Figure 9 and 13. The recrystallization is probably enhanced by oxidation, but recystallization was also visible within the material which means that recrystallization is not only due to oxidation.

As twinning was found to be the major deformation mechanism for the CMSX-4 material subjected to OP TMF testing in the $<001>$ direction, this paper strengthens previous work by Moverare et al. [4]. Extensive twinning was also found for the material loaded in $<011>$ crystallographic direction at low strain ranges. A deviation from this behavior was found for the material loaded in the $<011\rangle$ direction at high strain ranges. In this case the microstructure was more sheared and subsequently recrystallization occurred within the sheared deformation bands. The previous research [4] also discussed that crack propagation is a 
consequence of recrystallization and not the other way around. The findings in this new research confirm this behavior. It is clearly visible in Figure 15(a)-(d) that a crack has initiated in a recrystallized deformation band. The type of sheared deformation band in Figure 15(b) was previously reported for a high-Cr single-crystal superalloy exposed to OP TMF in the $<001>$ direction $[17]$.

Figure 4 show an unexpected result where the material tested in the $<011>$ direction showed a decreasing peak stress at half life up to 600 cycles to failure but subsequently the peak stress was increased. This abnormal behavior can be explained by the observed differences in deformation mechanisms. For low strain ranges, i.e. a high number of cycles to failure, twinning was found to be the major deformation mechanism. Specimens subjected to high strain ranges instead showed shearing of the $\gamma / \gamma^{\prime}$-microstructure as major deformation mechanism. In the case when twinning is dominant, the twins cut through the strengthening $\gamma^{\prime}$-particles, but in this case the $\gamma^{\prime}$-particles can still provide strength to the material. On the other hand, when shearing is dominant, the $\gamma^{\prime}$-particles are sheared apart leading to a decrease in strength. If shearing leads to softening of the microstructure this explains why the specimens subjected to high strain ranges shows lower peak stress at half life than expected.

\section{Summary and Conclusions}

The following conclusions can be drawn:

- High strain ranges results in crystallographic fractures along on of the $\{111\}$ planes while low strain ranges lead to non-crystallographic fractures. This is valid for both $<001>$ and $<011>$ crystallographic directions.

- The deformation is very localized for both crystallographic directions.

- TCP formation occurs frequently along the deformation bands.

- Signs of recrystallization can be found within the deformation bands.

- For the $<011>$ crystallographic direction a change in deformation mechanism from twinning to shearing of the microstructure is observed when going from low to high mechanical strain ranges.

- Mechanical damage such as twinning and shearing have the major influence on OP TMF endurance limit while oxidation has a minor influence. This is valid for OP TMF with $T_{\max }$ at $850^{\circ} \mathrm{C}$.

\section{Acknowledgements}

The work has been financially supported by Siemens Industrial Turbomachinery AB in Sweden and the Swedish Energy Agency, via the Research Consortium of Materials Technology for Thermal Energy Processes, Grant No. KME-502.

\section{References}

1. R.C. Reed, T. Tao, and N. Warnken, "AlloysBy-Design: Application to nickel-based single-crystal superalloys," Acta Materialia, 57 (2009), 5898-5913.

2. T. M. Pollock and S. Tin, "Nickel-Based Superalloys for Advanced Turbine Engines: Chemistry, Microstructure, and Properties," Journal of Propulsion and Power, 22 (2006), 361-374.

3. R.C. Reed, The Superalloys - Fundamentals and Applications (Cambridge: Cambridge University Press, 2006).

4. J.J. Moverare, S. Johansson, and R.C. Reed, "Deformation and damage mechanisms during thermalmechanical fatigue of a single-crystal superalloy," Acta Materialia, 57 (2009), 2266-2276.

5. J.X. Zhang, H. Harada, Y. Ro, Y. Koizumi, and T. Kobayashi, "Thermomechanical fatigue mechanism in a modern single-crystal nickel base superalloy, " Acta Materialia, 56 (2008), 2975-2987.

6. J.X. Zhang, Y. Ro, H. Zhou, and H. Harada, "Deformation twins and failure due to thermomechanical cycling in TMS-75 superalloy," Scripta Materialia, 54 (2006), 655-660.

7. M. Okazaki and M. Sakaguchi, "Thermo-mechanical fatigue failure of a single crystal Ni-based superalloy," International Journal of Fatigue, 30 (2008), 318-323.

8. L.N. Wang, Y. Liu, J.J. Yu, Y. Xu, X.F. Sun, H.R. Guan, and Z.Q. Hu, "Orientation and temperature dependence of yielding and deformation behavior of a nickel-base single crystal superalloy," Materials Science and Engineering A, 505 (2009), 144-150.

9. E.F. Westbrooke, L.E. Forero, and F. Ebrahimi, "Slip analysis in a Ni-base superalloy," Acta Materialia, 53 (2005), 2137-2147.

10. J. Courbon, M. Ignat, and F. Louchet, "Compression creep of $<110>$-oriented single crystals of 
nickel-base superalloy CMSX-2," Acta Metallurgica et Materialia, 38 (1990), 663-670.

11. M. Feller-Kniepmeier and T. Kuttner, "[011] creep in a single crystal nickel base superalloy at 1033 K," Acta Metallurgica et Materialia, 42 (1994), 3167-3174.

12. C. Buque, "Dislocation structures and cyclic behaviour of [011] and [111] oriented nickel single crystals," International Journal of Fatigue, 23 (2001), 671-678.

13. P. Caron, D. Cornu, T. Khan, and J.M de Monicault, "Development of a hydrogen resistant superalloy for single crystal blade application in rocket engine turbopumps," Superalloys 1996, ed. R.D. Kissinger et al. (Warrendale, PA: The Minerals, Metals and Materials Society, 1996), 53-60.

14. H. Mughrabi, "Microstructural aspects of high temperature deformation of monocrystalline nickel base superalloys: some open problems," Materials Science and Technology, 25 (2009), 191-204.

15. G.P. Zhang and Z.G. Wang, "Crystallographic cracking behavior in $\langle 110\rangle$ oriented $\mathrm{Ni}_{3} \mathrm{Al}$ alloy single crystals under cyclic loading," Materials Letters, 30 (1997), 175-181.

16. H.U. Hong, J.G. Kang, B.G. Choi, I.S. Kim, Y.S. Yoo, and C.Y. Jo, "A comparative study on thermomechanical and low cycle fatigue failures of a single crystal nickel-based superalloy," International Journal of Fatigue, 33 (2011), 1592-1599.

17. J.J. Moverare and S. Johansson, "Damage mechanisms of a high-Cr single crystal superalloy during thermomechanical fatigue," Materials Science and Engineering A, 527 (2010), 553-558. 